\title{
Neuroferritinopathy: iron in the brain
}

John Burn

From International Conference on Human Genetics and 39th Annual Meeting of the Indian Society of
Human Genetics (ISHG)
Ahmadabad, India. 23-25 January 2013

Careful attention to clinical phenotypes can identify new diseases which are now amenable to molecular genetic elucidation. In the late 1980's I met a family labelled as having Huntington's disease but with absence of dementia. Scanning revealed unusual cavitation of the basal ganglia. The pedigree was extended my connection to a second family using birth records. We mapped the gene to chromosome 19 and went on to identify an unusual mutation in the E helix of light chain ferritin. Staining for iron revealed huge accumulation of iron/ferritin complexes in the brain leading to neurodegeneration. We named the condition neuroferritinopathy and tested desferrioxamine without success. We have shown that accumulation of iron commences in childhood and are now preparing to test deferiprone as an iron chelator which crosses the blood brain barrier.

Published: 21 January 2014

doi:10.1186/1755-8166-7-S1-136

Cite this article as: Burn: Neuroferritinopathy: iron in the brain.

Molecular Cytogenetics 2014 7(Suppl 1):136.

Correspondence: john.burn@newcastle.ac.uk

Institute of Genetic Medicine, Newcastle University, International Centre for Life, Newcastle upon Tyne, UK any medium, provided the original work is properly cited. The Creative Commons Public Domain Dedication waiver (http:// creativecommons.org/publicdomain/zero/1.0/) applies to the data made available in this article, unless otherwise stated. 